\title{
Hyperprogression after immunotherapy
}

\author{
Waseem Abbas, Ranga Raju Rao, Swati Popli
}

\begin{abstract}
Introduction: Checkpoint inhibitors demonstrate very good anticancer effects, and some patients are long-time responders. As our experience to use these drugs increases, we see more and more patients having different kind of side effects which are usually not seen with chemotherapy. We have observed a subset of patients who appear to be "hyper-progressors," with a greatly accelerated rate of tumor growth and clinical deterioration compared to pretherapy, which was also recently reported by Institut Gustave Roussy. Materials and Methods: Medical records from all patients $(N=50)$ prospectively treated in our hospital by anti-PD-I/PD-LI were analyzed. The tumor growth rate (TGR) prior ("REFERENCE;" REF) and upon ("EXPERIMENTAL"; EXP) anti-PD-I/ PD-LI therapy was compared to identify patients with accelerated tumor growth. Associations between TGR and overall survival (OS) were computed. Results: Hyperprogressive disease (HPD) was defined as a RECIST progression at the first evaluation and as a $\geq 2$-fold increase of the TGR between the REF and the EXP periods. Of 50 evaluable patients, four patients (8\%) were considered as having HPD. At progression, patients with HPD had a higher rate of new lesions. HPD was associated with a worse outcome (OS). Conclusion: Hyperprogression was seen in 4 of 50 (8\%) of patients, three of which had urothelial cancer and one malignant melanoma, treated with anti-PD-I or anti-PD-LI monotherapy. Patients, on immunotherapy, qualifying for hyperprogression had shorted OS. It is important to have a better understanding of hyperprogression on immunotherapy which shall be addressed in the ongoing immunotherapy studies.
\end{abstract}

Key words: Hyperprogression after immunotehrapy, hyperprogression with check point inhibitors, immunotherapy linked to hyperprogression

\section{Introduction}

Immune checkpoint inhibitors are becoming standard of care for multiple cancer types. As experience grows with this therapeutics, anecdotal reports started relating rapid disease progressions, which could suggest that immune checkpoint blockade may have a deleterious effect by accelerating the disease in a subset of patients. Champiat et al. ${ }^{[1]}$ defined the hyperprogressive disease (HPD) as a $\geq 2$-fold increase of the tumor growth rate (TGR) between the REF and the EXP periods. Twelve patients (9\%) were considered as having HPD. HPD was neither associated with higher tumor burden at baseline nor with any specific tumor type. At progression, patients with HPD had a lower rate of new lesions than patients with disease progression without HPD $(P<0.05)$. HPD is associated with a higher age $(P<0.05)$ and a worse outcome (overall survival [OS]). Saâda-Bouzid et al. ${ }^{[2]}$ defined hyperprogression in recurrent and/or metastatic Head and Neck Squamous Cell Carcinoma (HNSCC), treated with PD-1/PD-L1 inhibitors, as tumor growth kinetics $\mathrm{R} \geq 2$. Hyperprogression was observed in $29 \%$ of patients with R/M HNSCC treated with anti-PD-L1/PD-1 agents and correlated with a shorter progression-free survival. Kato et al..$^{[3]}$ defined hyperprogression as time-to-treatment failure (TTF) $<2$ months, $>50 \%$ increase in tumor burden compared to preimmunotherapy imaging, and $>2$-fold increase in progression pace. After anti-PD-1/PD-L1 monotherapy, four of these patients showed remarkable increases in existing tumor size (55\%-258\%), new large masses, and significantly accelerated progression pace. Ferrara et al. ${ }^{[4]}$ defined HPD as disease progression at the first evaluation with $\Delta$ TGR exceeding $50 \%$. Fifty-six patients $(13.8 \%)$ were classified as having HPD. HPD was significantly associated with more than two metastatic sites before PD-1/PD-L1 inhibitors compared with non-HPD (62.5\% [35 of 56] vs. 42.6\% [149 of 350]; $P=0.006)$.

\section{Materials and Methods}

We did a retrospective analysis of fifty patients treated with immunotherapy for different malignancies at our center

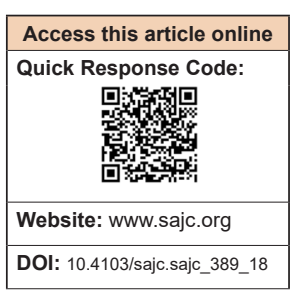

and identified a unique subset of patients whose disease paradoxically accelerated on immunotherapy. Herein, we describe our cohort of hyperprogressors.

We defined hyperprogression as 2-fold increase in tumor size. Time to failure $<2$ months was not used because the first evaluation for majority of patients was done after 2 months. TGR EXP/TGR Ratio $\geq 2$ also was not used because of its complexity.

To explore the prevalence of a potential HPD phenomenon in patients with cancer treated by anti-PD-1/PD-L1, we sought to compare TGRs of tumors during REFERENCE (i.e., before treatment onset; REF) and EXPERIMENTAL (i.e., between baseline and the first tumor evaluation; EXP) treatment periods.

\section{Case \#1}

A 62-year-old male was diagnosed with low-grade transitional cell carcinoma (TCC) in 2011 and then high-grade TCC in 2016 and underwent neoadjuvant chemotherapy (NACT) with three cycles of gemcitabine and cisplatin, followed by robotic radical cystectomy (ypT1N1) and three more cycles of adjuvant gemcitabine and cisplatin. After disease free survival of 1 year, disease relapsed in lungs. Patient was started on second-line paclitaxel and carboplatin for three cycles and assessment done revealed progression in lung with $25 \%$ increase in lesion. The patient was started on nivolumab, and after four doses, the patient developed multiple bone metastases including phalanges. In addition, imaging showed 200\% increase in lung lesion with increase in breathlessness and weight loss, which qualified for hyper progression Palliative radiation was given to painful bone metastatic sites [Figures 1a and 2].

\section{Case \#2}

A 68-year-old male was diagnosed with high-grade urothelial carcinoma in February 2017 and underwent concurrent chemoradiotherapy with cisplatin till March 2017 and was in complete response and on follow-up in October 2017 revealed multiple livers and lung metastases. After progressing on

This is an open access journal, and articles are distributed under the terms of the Creative Commons Attribution-NonCommercial-ShareAlike 4.0 License, which allows others to remix, tweak, and build upon the work non-commercially, as long as appropriate credit is given and the new creations are licensed under the identical terms.

For reprints contact: reprints@ medknow.com

How to cite this article: Abbas W, Rao RR, Popli S. Hyperprogression after immunotherapy. South Asian J Cancer 2019;8:244-6.
Department of Medical Oncology, MAX Institute of Cancer Care, Shalimarbagh, Delhi, India E-mail:drabbasdoc@gmail.com 
chemotherapy, he was started on nivolumab from December 2017 to January 2018 and received three cycles. He had weight loss, and repeat positron emission tomography-computed tomography (PET-CT) for assessment revealed new liver and lung lesions and $400 \%$ increase as per RECIST. Furthermore, pleural effusion was a new finding and qualified for hyperprogression [Figures $1 \mathrm{~b}$ and 2].

\section{Case \#3}

A 60 -year-old male was diagnosed with high-grade urothelial carcinoma of the left ureter in August 2015 and received four cycles of NACT with gemcitabine and carboplatin and underwent surgery (left nephrectomy and uretrectomy). Circadian rhythm-modulated chemotherapy was involved and received adjuvant radiation, followed by chemotherapy with gemcitabine and carboplatin for two cycles and he relapsed in January 2018 at local site with disease infiltrating into spinal vertebra and received palliative radiation along with pemrolizumab for six cycles until June 23, 2018. Assessment done after six cycles of immunotherapy showed 200\% increase in tumor burden as per RECIST and multiple new liver lesions qualifying for hyperprogression [Figures 1c and 2].

\section{Case \#4}

A 42-year-old female diagnosed with B-RAF and C-KIT mutation negative malignant melanoma in 2009. She received adjuvant interferon A till June 2009 and relapsed at the left buttock and she underwent surgery for primary and left inguinal lymph node dissection ( 2 out of 14 lymph nodes were positive). She received adjuvant radiation and relapsed in lung in 2017 and underwent video-assisted thoracoscopic surgery and wedge resection of left lung lower lobe. There was no adjuvant treatment and relapsed in left lung in December 2017 and was started on pembrolizumab from March 2018 to August 2018 for six cycles. PET-CT done showed $200 \%$ increase in lung lesion and multiple new bone and liver lesions qualifying for hyperprogression [Figures 1d and 2].

\section{Results}

We analyzed a total of 50 patients treated with anti-PD-1 or anti-PD-L1 monotherapy. Patients without a baseline CT scan at the start of immunotherapy were excluded from the study. All three patients with urothelial carcinoma were on chemotherapy before the start of immunotherapy, and the patient with malignant melanoma was on follow-up. We compared TGR of tumor before starting immunotherapy for TGR during both the REF periods (i.e., most often, between the imaging examination indicating prior progression and baseline) and the EXP periods. All four cases qualified for hyperprogression as they have twofold increase in size and plus what we observed also they developed multiple bone and liver metastasis and very high tumor burden at the time of assessment. When we looked into survival data, there was a clear trend toward worse outcome for the patients with HPD (median OS, 3 months) [Figure 3].

\section{Discussion}

For the first time ever, oncologists now face drugs with an extraordinary potential, but which also may induce a dramatic tumor surge in a fraction of patients. Overall, the HPD phenomenon with immune checkpoint blockade appears to be restricted to a small group of patients $(\sim 10 \%)$. We have seen in earlier studies with adjuvant interferon used for malignant

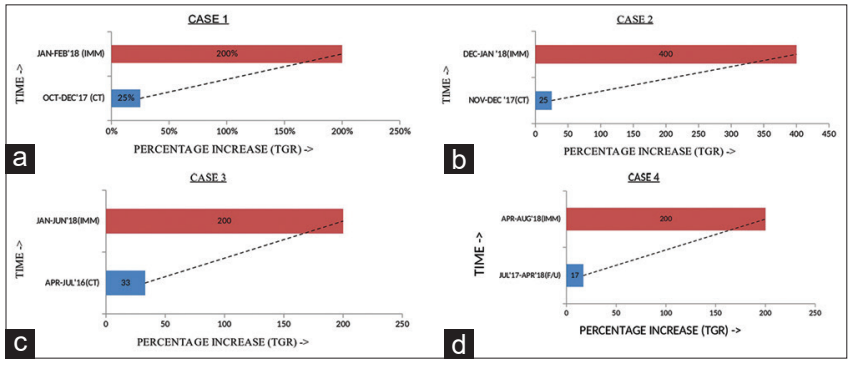

Figure 1: (a) Tumor growth rate on chemotherapy and immunotherapy, (b) tumor growth rate on chemotherapy and immunotherapy, (c) tumor growth rate on chemotherapy and immunotherapy, (d) tumor growth rate on chemotherapy and immunotherapy

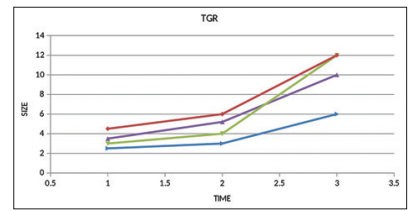

Figure 2: Tumor growth rate on chemotherapy and immunotherapy

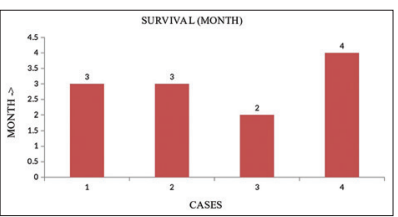

Figure 3: Association between hyperprogressive disease and overall survival melanoma. Those patients who died had a very reduced time from relapse to death. ${ }^{[5]}$ Hence, the phenomenon of disease progression is not only associated with immunotherapy but also with other therapeutic agents as well. ${ }^{[6,7]}$ There could be oncogenic signal activation, and PD-1/PD-L1signalling has cell-intrinsic factors.

The striking acceleration of tumor disease observed in patients with HPD could suggest an oncogenic signaling activation. ${ }^{[8]}$ It has been demonstrated that PD-1/PD-L1 signaling has cell-intrinsic functions in tumor cells. Immune compensatory mechanisms may play a role, and upregulation of alternative immune system can activate protumor immune subsets. ${ }^{[9,10]}$

We defined hyperprogression as $>$ twofold increase in progression pace. We did not take hyperprogression as $\mathrm{TTF}<2$ months into consideration because evaluation was done beyond 2 months for some patients.

In this study, four patients qualified for hyperprogression. Of four patients, three patients had urothelial carcinoma and one patient had malignant melanoma.

Chiampiat et al. showed patients with HPD exhibited a lower rate of new lesions than patients with non-HPD progression. Our study showed that apart from progression at primary site, there was florid progression at other sites as well, especially liver and bones.

One can always debate that in all the above-mentioned studies, ${ }^{[1-4]}$ immunotherapy was started as a treatment option in third-line or fourth-line settings, and this is the time where disease starts progressing very fast and the patients have few months to live. The biggest question remains, is this the natural history of disease or hyperprogression? Now, as checkpoint inhibitors are being used in first-line setting, we will have better understanding of hyperprogression. This question shall be addressed in the ongoing immunotherapy studies.

\section{Declaration of patient consent}

The authors certify that they have obtained all appropriate patient consent forms. In the form the patient(s) has/have given his/her/their consent for his/her/their images and other clinical information to be reported in the journal. The patients 
understand that their names and initials will not be published and due efforts will be made to conceal their identity, but anonymity cannot be guaranteed.

\section{Acknowledgment}

I am thankful to my coordinators with their support this work was not possible (Neha Goel, Mansi Jain, Virender).

\section{Financial support and sponsorship}

Nil.

\section{Conflicts of interest}

There are no conflicts of interest.

\section{References}

1. Champiat S, Dercle L, Ammari S, Massard C, Hollebecque A, Postel-Vinay $\mathrm{S}$, et al. Hyperprogressive disease is a new pattern of progression in cancer patients treated by anti-PD-1/PD-L 1. Clin Cancer Res 2017;23:1920-8.

2. Saâda-Bouzid E, Defaucheux C, Karabajakian A, Coloma VP, Servois V, Paoletti $X$, et al. Hyperprogression during anti-PD-1/PD-L1 therapy in patients with recurrent and/or metastatic head and neck squamous cell carcinoma. Ann Oncol 2017;28:1605-11.

3. Kato S, Goodman A, Walavalkar V, Barkauskas DA, Sharabi A, Kurzrock R, et al. Hyperprogressors after immunotherapy: Analysis of genomic alterations associated with accelerated growth rate. Clin Cancer Res 2017;23:4242-50.

4. Ferrara R, Mezquita L, Texier M, Lahmar J, Audigier-Valette C, Tessonnier L, et al. Hyperprogressive disease in patients with advanced non-small cell lung cancer treated with PD-1/PD-L1 inhibitors or with single-agent chemotherapy. JAMA Oncol 2018;4:1543-52.

5. Strannegård Ö, Thorén FB. Opposing effects of immunotherapy in melanoma using multisubtype interferon-alpha-can tumor immune escape after immunotherapy accelerate disease progression? Oncoimmunology 2016;5:e 1091147.

6. Mellema WW, Burgers SA, Smit EF. Tumor flare after start of RAF inhibition in KRAS mutated NSCLC: A case report. Lung Cancer 2015;87:201-3.

7. Kuriyama $\mathrm{Y}$, Kim YH, Nagai H, Ozasa H, Sakamori $\mathrm{Y}$, Mishima M, et al. Disease flare after discontinuation of crizotinib in anaplastic lymphoma kinase-positive lung cancer. Case Rep Oncol 2013;6:430-3.

8. Kleffel S, Posch C, Barthel SR, Mueller H, Schlapbach C, Guenova E, et al. Melanoma cell-intrinsic PD-1 receptor functions promote tumor growth. Cell 2015;162:1242-56

9. Francisco LM, Sage PT, Sharpe AH. The PD-1 pathway in tolerance and autoimmunity. Immunol Rev 2010;236:219-42.

10. Koyama S, Akbay EA, Li YY, Herter-Sprie GS, Buczkowski KA, Richards WG, et al. Adaptive resistance to therapeutic PD-1 blockade is associated with upregulation of alternative immune checkpoints. Nat Commun 2016;7:10501. 